\title{
Long-term treatment-related morbidity in differentiated thyroid cancer: a systematic review of the literature
}

This article was published in the following Dove Press journal:

Pragmatic and Observational Research

16 May 2017

Number of times this article has been viewed

\author{
William AE Parker ${ }^{1,2}$ \\ Ovie Edafe ${ }^{3}$ \\ Sabapathy P \\ Balasubramanian ${ }^{1,3}$ \\ 'Department of Oncology and \\ Metabolism, ${ }^{2}$ Department of Infection, \\ Immunity and Cardiovascular Disease, \\ The Medical School, The University \\ of Sheffield, ${ }^{3}$ Endocrine Surgery Unit, \\ Directorate of General Surgery, \\ Royal Hallamshire Hospital, Sheffield \\ Teaching Hospitals NHS Foundation \\ Trust, Sheffield, UK
}

Background: Differentiated thyroid cancer (DTC) occurs in relatively young patients and is associated with a good prognosis and long survival. The management of this disease involves thyroidectomy, radioiodine therapy, and long-term thyroid-stimulating hormone suppression therapy (THST). The long-term effects of the treatment and the interaction between subclinical hyperthyroidism and long-term hypoparathyroidism are poorly understood. This review sought to examine the available evidence.

Methods: A PubMed search was carried out using the search terms "Thyroid Neoplasms" AND ("Thyroxine" OR "Hypocalcemia" OR "Thyrotropin"). Original English language articles published in the last 30 years studying the morbidity from thyroid-stimulating hormone (TSH) suppression and hypoparathyroidism following a surgery for DTC were retrieved and reviewed by 2 authors.

Results: Of the 3,000 results, 66 papers including 4,517 patients were selected for the present study. Studies reported on a range of skeletal (included in 34 studies, 1,647 patients), cardiovascular (17 studies, 957 patients), psychological (10 studies, 663 patients), and other outcomes (10 studies, 1,348 patients). Nine of 26 studies on patients who underwent THST showed a reduction in bone density, and 13 of 23 studies showed an increase in bone turnover markers. Skeletal effects were more marked in postmenopausal women. There was no evidence of increased fracture risk, and only little data were available on hypoparathyroidism. Four of five studies showed an increased left ventricular mass index on echocardiography, and one study showed a higher prevalence of atrial fibrillation (AF). There was little difference in basic physiological parameters and limited literature regarding symptoms or significant events. Six studies showed associations between long-term TSH suppression and impaired quality of life. Impaired glucose metabolism and prothrombotic states were also found in DTC patients.

Conclusion: There is limited literature regarding long-term DTC treatment-related morbidity, particularly regarding the effects of long-term hypocalcemia. Most studies have focused on surrogate markers and not on clinical outcomes. A large prospective study on defined clinical outcomes would help characterize the morbidity of treatment and stimulate research on tailoring treatment strategies.

Keywords: neoplasia, clinical, bone, cardiovascular, surgery, adult thyroid, general

\section{Introduction}

Differentiated thyroid cancer (DTC) is the most common form of thyroid cancer. It includes papillary (80\%) and follicular (15\%) subtypes, as well as a small proportion of mixed tumors.

Women are more commonly affected by DTC than men with a ratio of $\sim 3: 1$. Age distribution is binomial with peaks between 35 and 39 years and $>70$ years. Almost half of the cases are diagnosed in patients aged $<50$ years. Despite the relatively young
Correspondence: William AE Parker Department of Infection, Immunity and Cardiovascular Disease, The Medical School, The University of Sheffield, Beech Hill Road, Sheffield SIO 2RX, UK

Tel +44 II4226 6I59

Email w.parker@sheffield.ac.uk 
average age, patients with DTC generally have a good prognosis of $\sim 90 \% 5$-year survival rate. ${ }^{1}$

In the last few decades, the incidence of thyroid cancer has increased 2.4-fold, higher than that of any other cancer among the US population. Greater diagnostic yield may play a role, but the incidence of cancers with a more aggressive subtype is also increasing. ${ }^{2}$ The standard treatments for DTC in the UK include total thyroidectomy (TT) for most patients with a tumor $>1 \mathrm{~cm}$ in size, radioactive iodine (RAI) ablation therapy for the majority of patients, and long-term thyroidstimulating hormone suppression therapy (THST). Practice across the world broadly includes the same strategy. ${ }^{3}$

The THST, by definition, leads to a state of at least subclinical hyperthyroidism, typically resulting in a low thyroid-stimulating hormone (TSH), high or high-normal thyroxine (T4) or free T4 (FT4), and a normal triiodothyronine (T3) or free $\mathrm{T} 3{ }^{3}$ This is in contrast with the typical profile in endogenous hyperthyroidism. Both endogenous hyperthyroidism and subclinical hyperthyroidism have long been linked to long-term adverse effects, particularly on the skeletal system. Thyroid hormone induces bone resorption via thyroid receptors TR $\alpha^{4}$ or TR $\beta^{5}$ on osteoclasts and osteoblasts. TSH may also have direct effects on bone metabolism. ${ }^{6}$ In addition, thyroid hormone also has well-characterized cardiovascular effects including increasing systolic blood pressure (BP), cardiac contractility, heart rate (HR), and tachyarrhythmias. ${ }^{7}$ Chronic endogenous hyperthyroidism can also lead to echocardiographic evidence of both systolic and diastolic dysfunctions in a significant proportion of patients. ${ }^{8}$

In addition, thyroidectomy for DTC may be accompanied by unintentional parathyroid damage or removal, resulting in postsurgical hypoparathyroidism. The latter may be transient, typically resolving shortly after surgery, or permanent, with potential long-term consequences. Hypoparathyroidism is known to reduce bone turnover and to increase bone mineral density (BMD) ${ }^{9}$ and can lead to metastatic calcification. ${ }^{10}$ This may be exacerbated by the additional calcium supplementation that is often required by these patients. ${ }^{11}$ Some studies have examined the incidence of postthyroidectomy hypocalcemia, ${ }^{12}$ but the long-term adverse effects of this treatment have not been well characterized in the DTC population. A recent study of patients with hypoparathyroidism following thyroidectomy for various indications demonstrated an increased tendency for infective and psychiatric illnesses and a reduced risk of fractures. ${ }^{13}$

There have been numerous small studies on DTC-related skeletal treatment-related morbidity (TRM), and these studies have been reviewed previously. ${ }^{14,15}$ However, more recently, a significant number of studies have been published, and there have been changes in surgical and endocrine practice, the assessment of BMD, biochemical measurement, and thyroxine dosing. There is also a lack of comprehensive review on the nonskeletal effects, and therefore, the present systematic review aimed to include up-to-date evidence of the long-term effects of DTC treatment on skeletal, cardiovascular, and other aspects of health.

\section{Review}

On June 28, 2014, the National Center for Biotechnology Information PubMed Central database was searched for articles using the Medical Subheading terms "Thyroid Neoplasms" AND ("Thyroxine" OR "Hypocalcemia" OR "Thyrotropin"). This search yielded 2,866 results, the abstracts of which were reviewed where applicable. All the original articles reporting on incidence, prevalence, and/or severity of morbidity relating to either postoperative hypocalcemia or TSH suppression in human patients who underwent treatment for DTC were considered for inclusion. Studies were excluded if they were published before 30 years; were not original studies (such as reviews, editorials, letters, and guidelines); did not include in vivo work on human subjects; were conducted on other populations or with no separate analyses of patients with DTC; involved nonstandard interventions; had outcomes not relevant to the research question (eg, short-term complications); were single-case reports; and were studies without a control group matched in some way with the population of interest. If the title and/or abstract of any study was unclear as to whether it should be included, the full text of that particular study was obtained for review (Figure 1). Then, the full texts of all the papers deemed suitable for inclusion were obtained, and the key data from these studies were recorded on a data-collection pro forma. Then, the data were transferred to an electronic database. The manuscripts were reviewed independently by 2 authors in order to validate the collected information. In total, 64 papers were included. The search was updated during the preparation of the manuscript on November 25, 2015 using the same search terms. A further 134 new abstracts were reviewed, from which two suitable papers were included. ${ }^{16,17}$

\section{Characteristics of the papers included}

In total, 66 original papers were included in the final analysis. These were mainly European studies, and a significant number of studies were published in the last decade (Figure 2). Thirty-four papers included skeletal outcomes, whereas 17, 10 and 10 papers included cardiovascular, psychological and other aspects, respectively. The present study reviewed for the presence of significant findings, those with a $p$-value $<0.05$. 


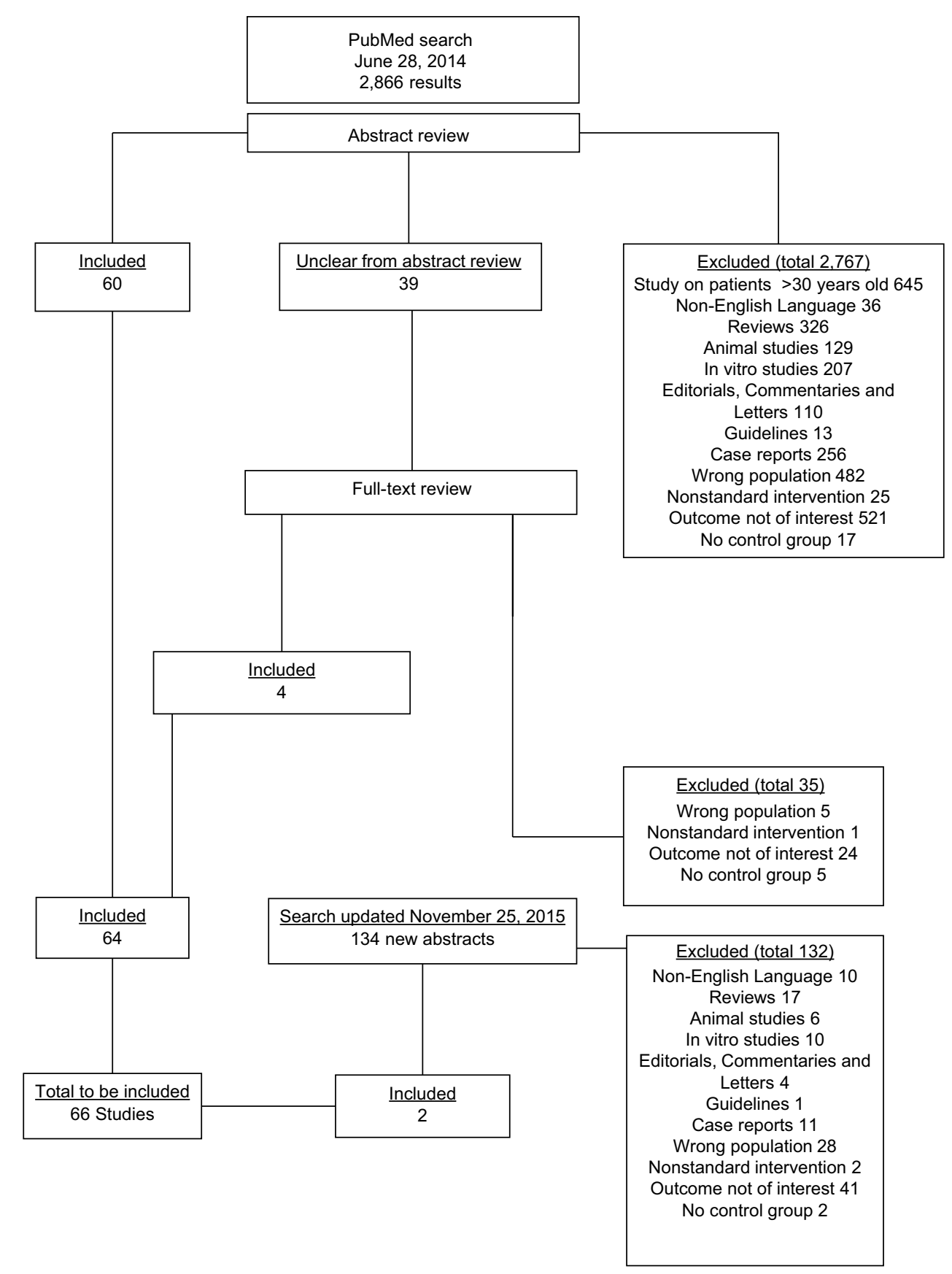

Figure I Flowchart of the systematic review process used to obtain relevant research articles.

\section{Summary}

\section{Skeletal effects}

Thirty-four papers included data on skeletal outcomes, with a total of 1,647 patients. Table S1 summarizes the characteristics of these studies. ${ }^{18-24}$ The majority of them were observational cross-sectional studies although there was a randomized controlled trial..$^{25}$ Some studies were primarily carried out to examine the effects of thyroid hormone withdrawal or the administration of recombinant TSH but also provided relevant data for inclusion in the present review. Two studies grouped all women together, ${ }^{25,26} 22$ studies reported on a separate premenopausal group, and 27 studies reported on a separate postmenopausal group. Twelve studies reported on a male cohort. The majority of study populations had undergone total or near TT followed by RAI and THST. In all but 6 studies, ${ }^{25,27-31}$ the control groups were comprised of healthy euthyroid-matched individuals or local reference populations. In 2 studies, ${ }^{28,30}$ the control group suffered from benign thyroid disease. Women with known osteoporosis suffering with DTC were compared with those with osteoporosis but no DTC in one case, ${ }^{29}$ and women with DTC and THST were compared with those with DTC but no THST (with the aim 

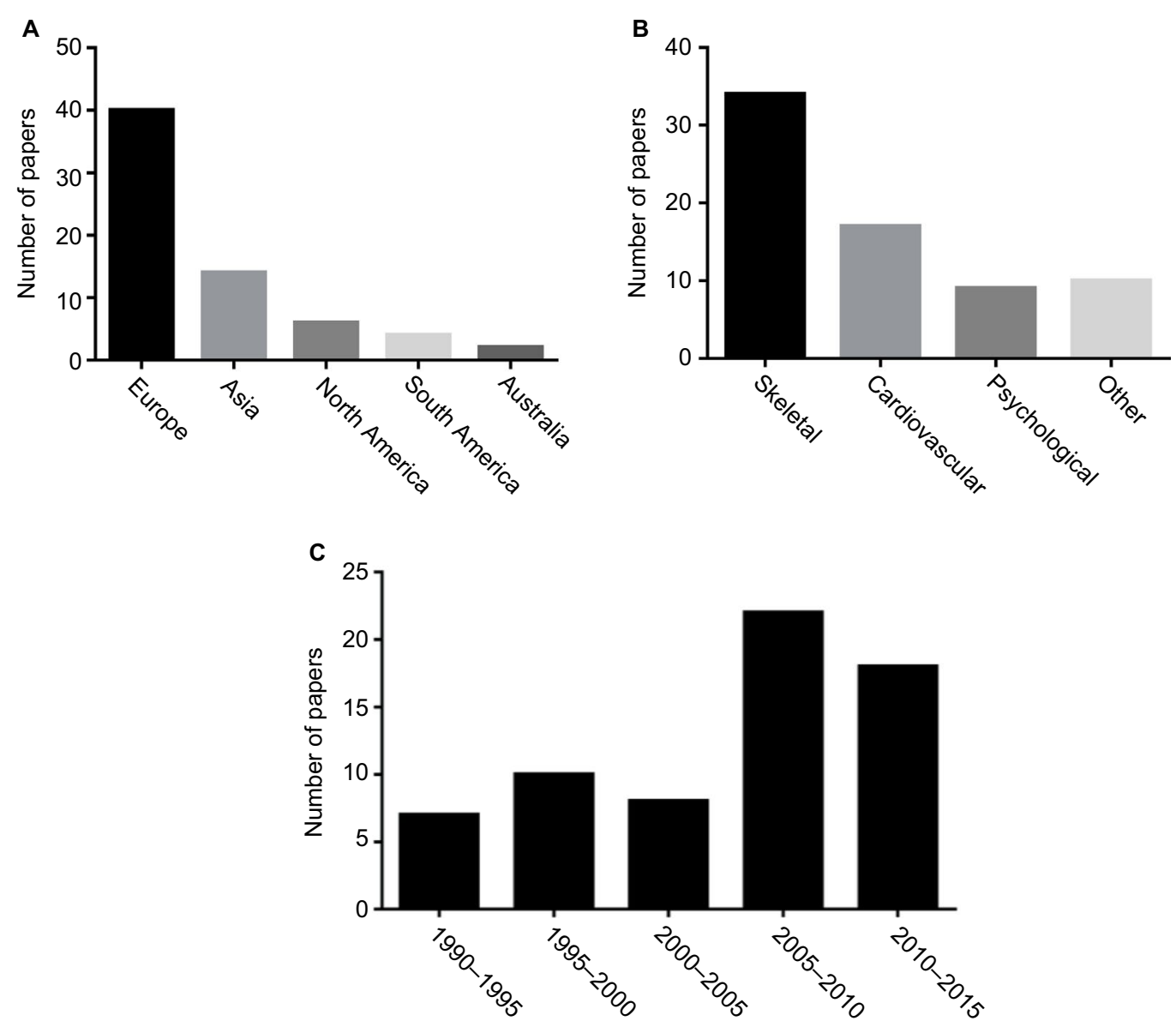

Figure 2 Characteristics of papers reporting on DTC treatment-related morbidity included in this systematic review, classified by (A) country of origin, (B) outcomes studied, and (C) year of publication.

Abbreviation: DTC, differentiated thyroid cancer.

of euthyroidism) in two cases. ${ }^{25,27}$ One study ${ }^{31}$ stratified DTC patients by parathyroid hormone levels ( 13 of 33 patients had hypoparathyroidism) and found increased BMD, reduced rate of BMD loss, and reduced bone turnover markers in hypoparathyroid versus normoparathyroid patients. Thirty-one studies either excluded patients with hypoparathyroidism or found no evidence of it in the studied population. In 26 studies, BMD was determined, and in 22 studies, bone turnover markers were measured. Other outcomes included the response of BMD to alendronate ${ }^{29}$ and the risk of low-impact fractures. ${ }^{32}$

\section{BMD}

Of the 26 studies that assessed the effect of THST in DTC patients on BMD, 9 showed some evidence of reduced BMD in patients on THST compared with controls. ${ }^{17,29,30,33-38}$ Table 1 summarizes the results of studies that compared BMD at common sites in premenopausal, postmenopausal, or male patients with DTC on THST against healthy controls.

Overall, 2 of 17 studies $^{34,38}$ that included a premenopausal female cohort and 5 of 21 studies $^{17,33-35,38}$ that included a postmenopausal female cohort demonstrated reduced BMD com- pared with healthy controls. Of the studies that included a control group patients with benign thyroid disease either on $\mathrm{THST}^{30}$ or in the euthyroid state, ${ }^{30}$ there was an evidence of reduced BMD (in the mid-radius) in the former, but not in the latter. Ten studies including a total of 136 male patients showed no significant differences in BMD when compared with control group. $26,32,39-46$

The one randomized controlled trial of THST versus "replacement only," carried out in Japan and included 144 female DTC patients, showed reduced BMD at 1 year in patients in their above 50s and at 5 years in all age-groups on THST. ${ }^{25}$ One paper that stratified patients by TSH found no significant differences in BMD between those with euthyroid and subclinical hyperthyroid status. ${ }^{27}$ Another paper that studied the effectiveness of alendronate in improving BMD found that BMD was reduced in DTC patients on THST compared with controls. ${ }^{29}$

\section{Bone turnover markers}

Twenty-three studies included the measurement of bone turnover markers, and 13 (57\%) studies showed an increase in bone turnover markers compared with controls, with no 
Table I Studies that included the measurement of bone mineral density in DTC patients on THST and healthy controls across various subgroups and sites

\begin{tabular}{|c|c|c|c|c|c|}
\hline Site & Group & Number of studies & $\begin{array}{l}\text { Total number } \\
\text { of patients }\end{array}$ & $\begin{array}{l}\text { Number of studies showing } \\
\text { significant reduction* }\end{array}$ & $\begin{array}{l}\text { Number of studies } \\
\text { showing no difference* }\end{array}$ \\
\hline \multirow[t]{3}{*}{ Lumbar spine } & Premenopausal women & 14 & 358 & 0 & 14 \\
\hline & Postmenopausal women & 13 & 366 & 3 & 10 \\
\hline & Men & 6 & 101 & 0 & 6 \\
\hline \multirow[t]{3}{*}{ Neck of femur } & Premenopausal women & 14 & 372 & 1 & 13 \\
\hline & Postmenopausal women & 14 & 346 & 3 & 11 \\
\hline & Men & 7 & 120 & 0 & 7 \\
\hline \multirow[t]{3}{*}{ Ward's triangle } & Premenopausal women & 6 & 143 & 0 & 6 \\
\hline & Postmenopausal women & 7 & 173 & 1 & 6 \\
\hline & Men & 2 & 23 & 0 & 2 \\
\hline \multirow[t]{3}{*}{ Trochanteric } & Premenopausal women & 6 & 132 & 0 & 6 \\
\hline & Postmenopausal women & 7 & 147 & I & 6 \\
\hline & Men & 3 & 42 & 0 & 3 \\
\hline
\end{tabular}

Notes: *In DTC groups compared with controls. $p<0.05$ was considered significant. No studies showed a significant increase in BMD in patients versus controls.

Abbreviations: BMD, bone mineral density; DTC, differentiated thyroid cancer; THST, thyroid-stimulating hormone suppression therapy.

difference in the remainder $(43 \%)$; in those studies that showed a difference, there was a range of different findings as summarized in Table 2.

\section{Other musculoskeletal outcomes}

Only one study assessed the risk of low-impact fracture as an outcome and found no difference between the patients and controls. ${ }^{32}$ Schneider et al studied the muscle strength using grip testing and showed no difference between male or premenopausal female patients and healthy controls. ${ }^{45}$

\section{Cardiovascular morbidity}

There were 14 studies related to cardiovascular morbidity and one study related to mortality. The latter study demonstrated a significant increase in both cardiovascular $(p<0.001)$ and all-cause $(p<0.001)$ mortality in a cohort of 524 patients with thyroid cancer compared with 1,572 controls over a median follow-up period of 8.5 years. ${ }^{47}$

The studies on cardiovascular morbidity included a range of physiological and pathological parameters (Table 3 ). The subjects included in these studies were patients treated for DTC with THST, and the controls were healthy, age- and sex-matched euthyroid individuals unless otherwise stated. Only one study included a measure of cardiovascular symptoms, and these symptoms were more prevalent in DTC patients than in a healthy control group. ${ }^{48}$ Table S2 summarizes the characteristics and key findings of the studies.

Table 2 Summary of the findings from studies of markers of bone turnover in patients with DTC on long-term THST

\begin{tabular}{|c|c|c|c|}
\hline Marker & Premenopausal women & Postmenopausal women & Men \\
\hline Alkaline phosphatase & No evidence of significant difference & Increased ${ }^{29,33,35,38}$ & Increased ${ }^{43}$ \\
\hline Osteocalcin & Increased ${ }^{38,46}$ & Increased $18,29,38$ & No evidence of significant difference \\
\hline Hydroxyproline & Increased 33,46 & Increased ${ }^{33,46}$ & No evidence of significant difference \\
\hline Type I collagen crosslinks & Increased $19,37,45,46$ & Increased $19,29,37,44,45$ & Increased $19-21,44$ \\
\hline Osteoprotegerin & Increased ${ }^{37}$ & Increased, ${ }^{37,43}$ reduced $^{28}$ & Increased, ${ }^{20}$ reduced $^{21}$ \\
\hline RANK-ligand & Increased, ${ }^{28}$ reduced $^{44}$ & Reduced $^{44}$ & Reduced $^{20,21,44}$ \\
\hline
\end{tabular}

Abbreviations: DTC, differentiated thyroid cancer; THST, thyroid-stimulating hormone suppression therapy; RANK, receptor activator of nuclear factor kappa-B.

Table 3 Summary of the findings from studies of long-term cardiovascular outcomes in patients with DTC

\begin{tabular}{llll}
\hline Variable & Number of studies & n(DTC) & Summary \\
\hline Heart rate & 9 & 170 & Increased resting heart rate in I of 9 studies \\
Blood pressure & 10 & 223 & Increased systolic and mean blood pressure in I of I0 studies \\
Prevalence of AF & 1 & 136 & Increased \\
LV mass & 6 & 116 & Increased in 4 of 6 studies \\
LV ejection fraction (systolic function) & 5 & 90 & No evidence of difference \\
Diastolic function & 4 & 74 & Evidence of higher markers of diastolic dysfunction in I of 4 studies \\
Cardiovascular mortality & 1 & 524 & Increased compared with controls \\
Response to exercise & $\mathrm{I}$ & 29 & Reduced VO \\
\hline
\end{tabular}

Abbreviations: AF, atrial fibrillation; DTC, differentiated thyroid cancer; LV, left ventricular; $\mathrm{VO}_{2 \text { max }}$ maximal oxygen consumption. 


\section{HR}

Nine studies reported on resting HR in a total of 170 DTC patients on THST and compared them against healthy controls. All but one study found no significant differences in resting HR between the patients and controls. ${ }^{48-55}$ One study showed an increase in HR compared with controls. ${ }^{56}$ Another study demonstrated no significant differences in minimum and maximum HRs, but a significant reduction in 1-minute HR variability in DTC patients. ${ }^{52}$ One study showed no significant differences between patients and controls in supine and standing HRs, but some evidence of reduced autonomic activity. ${ }^{51,57}$

\section{BP}

Ten studies studied BP in a total of 223 DTC patients on THST and 275 healthy controls. One study found a significantly higher mean and systolic BP in cancer patients, ${ }^{57}$ whereas others found no significant difference. No study found a significant difference in diastolic BP between both the groups. ${ }^{49-51,53-59}$ There were no significant differences in systemic vascular resistance in 2 studies..$^{50,56}$ One study demonstrated lower small and large artery elasticity in DTC patients on THST, ${ }^{56}$ whereas another study assessed brachial artery flow and glyceryl trinitrate-mediated dilatation and found no difference. ${ }^{59}$

\section{Arrhythmia}

The prevalence of AF in 136 DTC patients on THST over an 11-year period was compared with that of a population reference group and found to be elevated. ${ }^{60}$ Gen et al showed both increased maximum $\mathrm{P}$-wave duration and $\mathrm{P}$-wave dispersion in DTC patients compared with controls. ${ }^{61}$ However, there was no significant difference in the frequency of atrial or ventricular premature beats on Holter monitoring in another study ${ }^{48}$

\section{Echocardiography}

Echocardiographic parameters were measured in 6 studies. These studies included a total of 116 patients with DTC on THST and 130 healthy matched controls. There was a wide range of data on structural measurements. Five studies ${ }^{48,50,54-56}$ measured interventricular septal thickness. Two papers demonstrated this to be significantly increased in patients with DTC compared with controls, ${ }^{55,56}$ but no significant difference was found in the remainder. ${ }^{48,50,54}$ Left ventricular (LV) posterior wall thickness was found to be significantly increased in patients with DTC versus controls, ${ }^{55}$ but relative LV wall thickness was not different. ${ }^{53}$ In 4 of the 6 studies, LV mass index was shown to be significantly increased in patients with DTC compared with controls, ${ }^{48,54-56}$ but no difference was found in 2 studies. ${ }^{50,53}$ No paper demonstrated differences in LV end systolic diameter, LV end diastolic diameter, fractional shortening, LV ejection fraction, stroke volume, or cardiac output. Abdulrahman et al used speckle tracking echocardiography to assess LV strain in 14 DTC patients on THST and found a significant reduction in both circumferential and longitudinal but not radial strain, when compared with 24 controls. ${ }^{54}$

In addition to systolic function, 4 papers studied diastolic function. ${ }^{48,50,53,55}$ The early LV filling velocity was measured by using pulse wave Doppler $(E)$ imaging in all and found to be significantly reduced in DTC patients in two studies. ${ }^{53,55}$ The late ventricular filling velocity $(A)$ was not significantly different in controls, but despite this, there was no demonstrable significant difference in the $\mathrm{E} / \mathrm{A}$ ratio. The tissue Doppler imaging was able to demonstrate a significant reduction in $E^{\prime} / A^{\prime}$ in 14 DTC patients on THST for an average of 1 year, indicating a tendency toward diastolic dysfunction compared with controls. ${ }^{55}$ No differences in left atrial diameter were detected. In contrast, Taillard et al measured the isovolumetric relaxation and LV deceleration times and found no difference in the patients compared with controls..$^{53}$

\section{Response to exercise}

Vigario et al assessed response to cardiopulmonary exercise in 29 DTC patients who had been on THST for an average of 6 years and found a significant reduction in $\mathrm{VO}_{2 \max }$, workload, exercise intensity, peak HR, and HR variability in comparison with a group of 35 healthy matched controls. BP response to exercise and perception of breathlessness were not significantly different between the 2 groups. ${ }^{49}$

\section{Cognitive and psychological functions}

Jaracz et al assessed cognitive function in 31 patients on THST for DTC. ${ }^{62}$ They found that DTC patients made significantly more preservative and nonpreservative errors and completed fewer categories in the Wisconsin Card Sorting Test compared with age- and sex-matched healthy controls. In addition, these patients generated fewer words in the Oral Word Association Test and took longer time to complete Trail Making Tests. This suggested some impairment in executive functions and visuospatial working memory. No differences were found between the groups in scores of the Stroop Test and digital span, which also assesses executive functions, working memory, and attention.

In addition, Botella-Carretero et al also found some cognitive impairment in DTC patients on THST by using the WAIS digit span test. However, there was no difference in 
the other three cognitive tests used (ie, WAIS Digit Symbol, Davis Visual Scanning, and Cognitive Visual Analogical Mental Scales compared with controls). ${ }^{63}$

Moon et al did not find any differences in cognitive functions between 50 elderly DTC patients undergoing longterm TSH suppression compared with 90 matched healthy controls. Interestingly, in patients with DTC, higher levels of FT4 were associated with better scores in Mini Mental State Examination and Trail Making Test A. ${ }^{16}$

\section{Quality of life}

Botella-Carretero et al found that 18 DTC patients scored significantly worse on the Nottingham Health Profile and on general health, mental health, and social functions of the Short Form (SF)-36 Health Survey compared with healthy controls. ${ }^{63}$

Crevenna et al assessed the quality of life (using German version of SF-36) in 150 patients with nonmetastatic thyroid cancer on the long-term TSH suppression. Overall, patients scored worst in domains that assessed emotions, mood and energy levels, and limitation of activities due to emotional state (vitality and role emotions). In a subgroup analysis of patients assessed within 1 year of diagnosis of thyroid cancer, impairment was observed in more domains (mental health, role - emotional, role - physical, social functioning, and vitality) compared with reference values. Conversely, DTC patients scored better in items that evaluated general health and bodily pain. ${ }^{64}$

Eustatia-Rutten et al found that 24 patients with DTC undergoing long-term TSH suppression had significantly more somatic symptoms compared with reference values (Somatic Disorder Questionnaire [SDQ] total). However, they scored better on symptoms of low mood (depressive symptoms on Hospital Anxiety Depressive Score [HADS]). No significant difference was found in other quality of life parameters between the values of patients and reference values (SF-36, Multidimensional Fatigue Index-20 [MFI-20], Symptom Rating Scale, and HADS). ${ }^{65}$

Hoftijzer et al assessed the quality of life in 153 patients treated with TSH suppression followed by TT and radioiodine ablation for DTC. ${ }^{66}$ Using 4 validated questionnaires (ie, SF-36, MFI-20, HADS, and SDQ), patients were shown to have a significantly worse quality of life in at least 11 of 16 categories compared with controls. No association was found between serum TSH levels and quality of life. However, longer duration of cure from thyroid cancer was associated with improved quality of life.

Tagay et al evaluated the quality of life in 100 patients with DTC following TT, RAI, and TSH suppression. DTC patients scored significantly worse in all items of the SF-36 except for 1 item (bodily pain), when compared to German reference population. ${ }^{67}$ The total score of the mental components of the SF-36 was also significantly lower in DTC patients; however, there was no difference in physical component scores. In addition, the prevalence of anxiety and depressive symptoms (assessed by HADS, BDI, and Profile of Mood States) was within normal limits.

Vigario Pdos et al assessed fatigue perception, physical activity, and body composition in 36 patients with DTC following chronic THST. ${ }^{6}$ DTC patients had significantly smaller muscle mass and mid-thigh girth compared with healthy controls with sedentary lifestyles. There were no significant differences in body weight, body mass index, body fat, lean muscle mass, bone mass, residual mass, abdominal girth, and calf girth between the patients and controls. In addition, DTC patients reported significantly greater fatigue assessed on the Chalder fatigue scale, which assesses physical fatigue, tiredness, weakness, and muscle pain. The same groups were able to show that an exercise program improved their quality of life outcomes. ${ }^{69}$

\section{Glucose metabolism}

In a randomized controlled trial, Heemstra et al analyzed the effects of long-term TSH suppression on glucose and lipid metabolism in patients with DTC. ${ }^{70}$ At 6-month follow-up, there was no difference in glucose tolerance, insulin sensitivity, lipid profile, and glucoregulatory hormone levels between TSH-suppressed $(n=13)$ and euthyroid $(n=12)$ patients.

Hsieh et al examined the effect of hyperthyroidism on leptin concentration and body composition in patients with DTC. ${ }^{71}$ No significant difference was found in leptin concentration between euthyroid DTC patients and controls. However, no comparison was made between TSH-suppressed patients and controls.

Rezzonico et al assessed the association between insulin resistance and subclinical hyperthyroidism in 125 patients, ${ }^{72}$ 20 of whom had DTC. In subclinical hyperthyroidism, insulin and glucose were significantly higher at baseline and 2 hours after a standard meal. Insulin resistance (measured by homeostatic model assessment, the quantitative insulin sensitivity check index, and Avignon's index amended by Aloulou) was significantly higher in patients with subclinical hyperthyroidism compared with controls. However, there was no significant difference in the secretion of insulin by pancreatic $\beta$ cells between the patients and controls.

Mittal et al evaluated increases in cardiovascular risk in 50 patients with DTC. Serum glucose was significantly lower and insulin level was higher in DTC patients compared 
with controls, although treatment details were not fully described. ${ }^{58}$ However, there was no significant difference in glycated hemoglobin (HbA1c) levels. Total cholesterol, high-density lipoprotein, and low-density lipoprotein were significantly lower in DTC patients compared with controls, but no difference was found in triglycerides and very-lowdensity lipoprotein levels.

Table 4 summarizes the key findings of studies on cognitive function, quality of life, and glucose tolerance.

\section{Coagulation}

Horne et al examined the effects of chronic THST on coagulation in 14 patients following TT for DTC. ${ }^{73}$ Patients on THST had significantly higher levels of a range of coagulationrelated substances including prothrombin fragments 1 and 2, fibrinogen, factor VIII, antithrombin, tissue plasminogen activator (tPA), plasminogen activator inhibitor I (PAI-1), and PAI-1/tPA. Conversely, plasmin-antiplasmin complexes were lower. Other factors studied (plasminogen, factor II, and d-dimer) did not significantly change. Overall, the findings suggested a prothrombotic state during TSH suppression.

\section{Immunology}

In a study of 22 patients with DTC, Botella-Carretero et al found that TSH suppression significantly increased serum interleukin (IL)-18, soluble IL-2 receptor, and proportion of natural killer cells compared with euthyroid controls. ${ }^{74}$ The implications of these changes in patients with DTC are unclear.

\section{Conclusion}

A range of studies examining skeletal, cardiovascular, and a small number of other outcomes were studied in this review. There is some evidence that BMD is reduced, particularly in postmenopausal women treated for DTC compared with the general population, and there is also some evidence of significant differences in various echocardiographic parameters, notably markers of diastolic dysfunction, and of increased tendency for atrial arrhythmia.

Table 4 Summary of evidence for the effects of long-term TSH suppression in DTC patients on cognitive function, quality of life, and glucose tolerance

\begin{tabular}{lll}
\hline Variable & Evidence of reduction & Evidence of no effect \\
\hline Cognitive function & 2 of 3 studies $^{62,63}$ & I of 3 studies \\
Quality of life & 6 of 7 studies $^{63,64,66-69}$ & I of 7 studies $^{65}$ \\
Glucose tolerance & 2 of 3 studies $^{58,72}$ & I of 3 studies $^{70}$ \\
\hline
\end{tabular}

Abbreviations: DTC, differentiated thyroid cancer; TSH, thyroid-stimulating hormone.
It is important to clarify TRM in this population as a significant proportion of the adverse effects of DTC treatment are potentially preventable and/or reversible. For example, there is some evidence of the reversibility of diastolic dysfunction when THST is discontinued, ${ }^{75}$ and beta blockade has been shown to reverse some changes in the echocardiographic parameters and exercise tolerance in patients on THST for benign disease. ${ }^{76,77}$ It remains to be seen whether this might translate into meaningful benefits to the patients.

There are relatively few studies on TRM-related clinical outcomes in these patients. The relevance of the outcomes, assessed in the studies included in the present review, to the individual patient with DTC remains debatable. In particular, there is a paucity of studies examining fracture risk, major adverse cardiovascular events, and long-term TRM. Another key area requiring further work is the long-term effects of hypoparathyroidism, especially in DTC patients. Most of the studies reviewed either did not feature hypoparathyroid patients or excluded them. Although, in general, the prevalence of postthyroidectomy permanent hypoparathyroidism is felt to be low, in some studies it is as high as $12 \%$ and may be higher in procedures carried out for malignancy. There is evidence from benign disease that patients with hypoparathyroidism have increased BMD related to the general population, ${ }^{78}$ and therefore one might hypothesize that hypoparathyroidism in a patient on THST might offer some protection against the thyroxine-mediated loss of BMD. There is also some evidence that this translates into a reduced risk of fracture in these patients. ${ }^{13}$ However, this is controversial, and the patients may be affected by concurrent treatments such as calcium and vitamin D supplementation. In addition, although there may be a reduction in the loss of BMD, other dynamics of bone turnover may be affected, resulting in adverse outcomes. ${ }^{79}$

\section{Strengths}

The systematic search strategy has ensured that this review is comprehensive, as shown by the large number of papers retrieved in the original search. Only papers strictly relevant to the review question have been included, and studies of short-term outcomes were excluded as were those with nonstandard treatment interventions. Studies without clear methodology or not reflective of standard practice did not influence the evidence synthesis.

\section{Limitations}

The studies included had significant heterogeneity in terms of treatment regimes, target TSH levels, follow-up, and outcome 
variables, and this precluded the possibility of meaningful meta-analysis. The majority of studies, particularly those of nonskeletal outcomes, included only small numbers of patients. This highlights the need for studies with a larger number of patients. It is possible that some relevant studies might not have been included, especially those in non-English language literature; time and logistic considerations precluded their inclusion.

\section{Further work}

There appears to be sufficient evidence to highlight the potential of long-term TRM in patients with DTC to warrant further investigation on a larger and more systematic scale. A multicenter study of long-term skeletal, cardiovascular, and other outcomes including the quality of life in the DTC population would help to elucidate the adverse effects of treatment.

\section{Acknowledgments}

A poster of this work has previously been presented to the British Association of Thyroid and Endocrine Surgeons, Henley-on-Thames, UK, on October 2015, and to the 15th International Thyroid Congress, Orlando, USA, on October 2015.

\section{Disclosure}

Dr Parker reports an honorarium from Medscape. The other authors report no conflicts of interest in this work.

\section{References}

1. CRUK. Thyroid cancer incidence statistics; 2015. Available from: http://www.cancerresearchuk.org/health-professional/cancer-statistics/ statistics-by-cancer-type/thyroid-cancer/incidence. Accessed January 05, 2017.

2. Davies L, Welch HG. Increasing incidence of thyroid cancer in the United States, 1973-2002. JAMA. 2006;295(18):2164-2167.

3. McLeod DS, Sawka AM, Cooper DS. Controversies in primary treatment of low-risk papillary thyroid cancer. Lancet. 2013;381(9871): 1046-1057.

4. Bassett JH, O'Shea PJ, Sriskantharajah S, et al. Thyroid hormone excess rather than thyrotropin deficiency induces osteoporosis in hyperthyroidism. Mol Endocrinol. 2007;21(5):1095-1107.

5. Monfoulet LE, Rabier B, Dacquin R, et al. Thyroid hormone receptor $\beta$ mediates thyroid hormone effects on bone remodeling and bone mass. J Bone Miner Res. 2011;26(9):2036-2044.

6. Abe E, Marians RC, Yu W, et al. TSH is a negative regulator of skeletal remodeling. Cell. 2003;115(2):151-162.

7. Klein I, Ojamaa K. Thyroid hormone and the cardiovascular system. $N$ Engl J Med. 2001;344(7):501-509.

8. Ertek S, Cicero AF. Hyperthyroidism and cardiovascular complications: a narrative review on the basis of pathophysiology. Arch Med Sci. 2013;9(5):944-952.

9. Rubin MR, Bilezikian JP. Hypoparathyroidism: clinical features, skeletal microstructure and parathyroid hormone replacement. Arq Bras Endocrinol Metabol. 2010;54(2):220-226.
10. Goswami R, Sharma R, Sreenivas V, Gupta N, Ganapathy A, Das S. Prevalence and progression of basal ganglia calcification and its pathogenic mechanism in patients with idiopathic hypoparathyroidism. Clin Endocrinol. 2012;77(2):200-206.

11. Spence LA, Weaver CM. Calcium intake, vascular calcification, and vascular disease. Nutr Rev. 2013;71(1):15-22.

12. Edafe O, Antakia R, Laskar N, Uttley L, Balasubramanian SP. Systematic review and meta-analysis of predictors of post-thyroidectomy hypocalcaemia. Br J Surg. 2014;101(4):307-320.

13. Underbjerg L, Sikjaer T, Mosekilde L, Rejnmark L. Postsurgical hypoparathyroidism-risk of fractures, psychiatric diseases, cancer, cataract, and infections. J Bone Miner Res. 2014;29(11):2504-2510.

14. Quan ML, Pasieka JL, Rorstad O. Bone mineral density in well-differentiated thyroid cancer patients treated with suppressive thyroxine: a systematic overview of the literature. J Surg Oncol. 2002;79(1):62-70.

15. Heemstra KA, Hamdy NA, Romijn JA, Smit JW. The effects of thyrotropin-suppressive therapy on bone metabolism in patients with well-differentiated thyroid carcinoma. Thyroid. 2006;16(6):583-591.

16. Moon JH, Ahn S, Seo J, et al. The effect of long-term thyroid-stimulating hormone suppressive therapy on the cognitive function of elderly patients with differentiated thyroid carcinoma. JClin Endocrinol Metab. 2014;99(10):3782-3789.

17. Tournis S, Antoniou JD, Liakou CG, et al. Volumetric bone mineral density and bone geometry assessed by peripheral quantitative computed tomography in women with differentiated thyroid cancer under TSH suppression. Clin Endocrinol (Oxf). 2015;82(2):197-204.

18. Hawkins F, Rigopoulou D, Papapietro K, Lopez MB. Spinal bone mass after long-term treatment with L-thyroxine in postmenopausal women with thyroid cancer and chronic lymphocytic thyroiditis. Calcif Tissue Int. 1994;54(1):16-19.

19. Frusciante V, Carnevale V, Scillitani A, et al. Global skeletal uptake of technetium-99m methylene diphosphonate in female patients receiving suppressive doses of L-thyroxine for differentiated thyroid cancer. Eur J Nucl Med. 1998;25(2):139-143.

20. Mikosch P, Jauk B, Gallowitsch HJ, Pipam W, Kresnik E, Lind P. Suppressive levothyroxine therapy has no significant influence on bone degradation in women with thyroid carcinoma: a comparison with other disorders affecting bone metabolism. Thyroid. 2001;11(3):257-263.

21. Chen $\mathrm{CH}$, Chen JF, Yang BY et al. Bone mineral density in women receiving thyroxine suppressive therapy for differentiated thyroid carcinoma. J Formos Med Assoc. 2004;106(6):442-447.

22. Reverter JL, Holgado S, Alonso N, Salinas I, Granada ML, Sanmarti A. Lack of deleterious effect on bone mineral density of long-term thyroxine suppressive therapy for differentiated thyroid carcinoma. Endocr Relat Cancer. 2005;12(4):973-981.

23. Sajjinanont T, Rajchadara S, Sriassawaamorn N, Panichkul S. The comparative study of bone mineral density between premenopausal women receiving long term suppressive doses of levothyroxine for well-differentiated thyroid cancer with healthy premenopausal women. J Med Assoc Thai. 2005;88(Suppl 3):S71-S76.

24. Botella-Carretero JI, Alvarez-Blasco F, San Millan JL, EscobarMorreale HF. Thyroid hormone deficiency and postmenopausal status independently increase serum osteoprotegerin concentrations in women. Eur J Endocrinol. 2007;156(5):539-545.

25. Sugitani I, Fujimoto Y. Effect of postoperative thyrotropin suppressive therapy on bone mineral density in patients with papillary thyroid carcinoma: a prospective controlled study. Surgery. 2011;150(6):1250-1257.

26. Florkowski CM, Brownlie BE, Elliot JR, Ayling EM, Turner JG. Bone mineral density in patients receiving suppressive doses of thyroxine for thyroid carcinoma. N Z Med J. 1993;106(966):443-444.

27. Fujiyama K, Kiriyama T, Ito M, et al. Suppressive doses of thyroxine do not accelerate age-related bone loss in late postmenopausal women. Thyroid. 1995;5(1):13-17.

28. Giusti M, Cecoli F, Fazzuoli L, et al. Serum osteoprotegerin and soluble receptor activator of nuclear factor kappaB ligand levels in patients with a history of differentiated thyroid carcinoma: a case-controlled cohort study. Metabolism. 2007;56(5):699-707. 
29. Panico A, Lupoli GA, Fonderico F, et al. Osteoporosis and thyrotropinsuppressive therapy: reduced effectiveness of alendronate. Thyroid. 2009;19(5):437-442.

30. McDermott MT, Perloff JJ, Kidd GS. A longitudinal assessment of bone loss in women with levothyroxine-suppressed benign thyroid disease and thyroid cancer. Calcif Tissue Int. 1995;56(6):521-525.

31. Fujiyama K, Kiriyama T, Ito M, et al. Attenuation of postmenopausal high turnover bone loss in patients with hypoparathyroidism. $J$ Clin Endocrinol Metab. 1995;80(7):2135-2138.

32. Reverter JL, Colome E, Holgado S, et al. Bone mineral density and bone fracture in male patients receiving long-term suppressive levothyroxine treatment for differentiated thyroid carcinoma. Endocrine. 2010;37(3):467-472.

33. Giannini S, Nobile M, Sartori L, et al. Bone density and mineral metabolism in thyroidectomized patients treated with long-term L-thyroxine. Clin Sci (Lond). 1994;87(5):593-597.

34. Jodar E, Begona Lopez M, Garcia L, Rigopoulou D, Martinez G, Hawkins F. Bone changes in pre- and postmenopausal women with thyroid cancer on levothyroxine therapy: evolution of axial and appendicular bone mass. Osteoporos Int. 1998;8(4):311-316.

35. Kung AW, Lorentz T, Tam SC. Thyroxine suppressive therapy decreases bone mineral density in post-menopausal women. Clin Endocrinol. 1993;39(5):535-540.

36. Muller CG, Bayley TA, Harrison JE, Tsang R. Possible limited bone loss with suppressive thyroxine therapy is unlikely to have clinical relevance. Thyroid. 1995;5(2):81-87.

37. Mazziotti G, Sorvillo F, Piscopo M, et al. Recombinant human TSH modulates in vivo C-telopeptides of type-1 collagen and bone alkaline phosphatase, but not osteoprotegerin production in postmenopausal women monitored for differentiated thyroid carcinoma. $J$ Bone Miner Res. 2005;20(3):480-486.

38. Diamond T, Nery L, Hales I. A therapeutic dilemma: suppressive doses of thyroxine significantly reduce bone mineral measurements in both premenopausal and postmenopausal women with thyroid carcinoma. J Clin Endocrinol Metab. 1991;72(6):1184-1188.

39. Eftekhari M, Asadollahi A, Beiki D, et al. The long term effect of levothyroxine on bone mineral density in patients with well differentiated thyroid carcinoma after treatment. Hell J Nucl Med. 2008;11(3):160-163.

40. Franklyn JA, Betteridge J, Daykin J, et al. Long-term thyroxine treatment and bone mineral density. Lancet. 1992;340(8810):9-13.

41. Gorres G, Kaim A, Otte A, Gotze M, Muller-Brand J. Bone mineral density in patients receiving suppressive doses of thyroxine for differentiated thyroid carcinoma. Eur J Nucl Med. 1996;23(6):690-692.

42. Guo CY, Weetman AP, Eastell R. Longitudinal changes of bone mineral density and bone turnover in postmenopausal women on thyroxine. Clin Endocrinol (Oxf). 1997;46(3):301-307.

43. Heijckmann AC, Huijberts MS, Geusens P, de Vries J, Menheere PP, Wolffenbuttel BH. Hip bone mineral density, bone turnover and risk of fracture in patients on long-term suppressive L-thyroxine therapy for differentiated thyroid carcinoma. Eur J Endocrinol. 2005;153(1):23-29.

44. Martini G, Gennari L, De Paola V, et al. The effects of recombinant TSH on bone turnover markers and serum osteoprotegerin and RANKL levels. Thyroid. 2008;18(4):455-460.

45. Schneider R, Schneider M, Reiners C, Schneider P. Effects of levothyroxine on bone mineral density, muscle force, and bone turnover markers: a cohort study. J Clin Endocrinol Metab. 2012;97(11):3926-3934.

46. Toivonen J, Tahtela R, Laitinen K, Risteli J, Valimaki MJ. Markers of bone turnover in patients with differentiated thyroid cancer with and following withdrawal of thyroxine suppressive therapy. Eur J Endocrinol. 1998;138(6):667-673

47. Klein Hesselink EN, Klein Hesselink MS, de Bock GH, et al. Long-term cardiovascular mortality in patients with differentiated thyroid carcinoma: an observational study. J Clin Oncol. 2013;31(32):4046-4053.

48. Shapiro LE, Sievert R, Ong L, et al. Minimal cardiac effects in asymptomatic athyreotic patients chronically treated with thyrotropin-suppressive doses of L-thyroxine. J Clin Endocrinol Metab. 1997;82(8):2592-2595.
49. Vigario Pdos S, Chachamovitz DS, Teixeira Pde F, Santos MA, Oliveira FP, Vaisman M. Impaired functional and hemodynamic response to graded exercise testing and its recovery in patients with subclinical hyperthyroidism. Arq Bras Endocrinol Metabol. 2011;55(3):203-212.

50. Botella-Carretero JI, Gomez-Bueno M, Barrios V, et al. Chronic thyrotropin-suppressive therapy with levothyroxine and short-term overt hypothyroidism after thyroxine withdrawal are associated with undesirable cardiovascular effects in patients with differentiated thyroid carcinoma. Endocr Relat Cancer. 2004;11(2):345-356.

51. Murialdo G, Casu M, Cappi C, et al. Effects of recombinant human thyrotropin on heart rate variability and blood pressure in patients on 1-thyroxine-suppressive therapy for differentiated thyroid carcinoma. Horm Res. 2005;64(2):100-106.

52. Shuvy M, Arbelle JE, Grosbard A, Katz A. A simple test of one minute heart rate variability during deep breathing for evaluation of sympatovagal imbalance in hyperthyroidism. Isr Med Assoc J. 2008;10(8-9):603-606.

53. Taillard V, Sardinoux M, Oudot C, et al. Early detection of isolated left ventricular diastolic dysfunction in high-risk differentiated thyroid carcinoma patients on TSH-suppressive therapy. Clin Endocrinol (Oxf). 2011;75(5):709-714.

54. Abdulrahman RM, Delgado V, Hoftijzer HC, et al. Both exogenous subclinical hyperthyroidism and short-term overt hypothyroidism affect myocardial strain in patients with differentiated thyroid carcinoma. Thyroid. 2011;21(5):471-476.

55. Hoftijzer HC, Bax JJ, Heemstra KA, et al. Short-term overt hypothyroidism induces discrete diastolic dysfunction in patients treated for differentiated thyroid carcinoma. Eur J Clin Invest. 2009;39(3): 204-210.

56. Shargorodsky M, Serov S, Gavish D, Leibovitz E, Harpaz D, Zimlichman R. Long-term thyrotropin-suppressive therapy with levothyroxine impairs small and large artery elasticity and increases left ventricular mass in patients with thyroid carcinoma. Thyroid. 2006;16(4):381-386.

57. Casu M, Cappi C, Patrone V, et al. Sympatho-vagal control of heart rate variability in patients treated with suppressive doses of $\mathrm{L}$-thyroxine for thyroid cancer. Eur J Endocrinol. 2005;152(6):819-824.

58. Mittal A, Poudel B, Pandeya DR, Gupta SP, Sathian B, Yadav SK. Metabolic changes enhance the cardiovascular risk with differentiated thyroid carcinoma-a case control study from Manipal Teaching Hospital of Nepal. Asian Pac J Cancer Prev. 2012;13(5):2335-2338.

59. Dardano A, Ghiadoni L, Plantinga Y, et al. Recombinant human thyrotropin reduces endothelium-dependent vasodilation in patients monitored for differentiated thyroid carcinoma. J Clin Endocrinol Metab. 2006;91(10):4175-4178.

60. Abonowara A, Quraishi A, Sapp JL, et al. Prevalence of atrial fibrillation in patients taking TSH suppression therapy for management of thyroid cancer. Clin Invest Med. 2012;35(3):E152-E156.

61. Gen R, Akbay E, Camsari A, Ozcan T. P-wave dispersion in endogenous and exogenous subclinical hyperthyroidism. J Endocrinol Invest. 2010;33(2):88-91.

62. Jaracz J, Kucharska A, Rajewska-Rager A, Lacka K. Cognitive functions and mood during chronic thyrotropin-suppressive therapy with L-thyroxine in patients with differentiated thyroid carcinoma. $J$ Endocrinol Invest. 2012;35(8):760-765.

63. Botella-Carretero JI, Galan JM, Caballero C, Sancho J, EscobarMorreale HF. Quality of life and psychometric functionality in patients with differentiated thyroid carcinoma. Endocr Relat Cancer. 2003;10(4):601-610.

64. Crevenna R, Zettinig G, Keilani M, et al. Quality of life in patients with non-metastatic differentiated thyroid cancer under thyroxine supplementation therapy. Suppor Care Cancer. 2003;11(9):597-603.

65. Eustatia-Rutten CF, Corssmit EP, Pereira AM, et al. Quality of life in long-term exogenous subclinical hyperthyroidism and the effects of restoration of euthyroidism, a randomized controlled trial. Clin Endocrinol. 2006;64(3):284-291.

66. Hoftijzer HC, Heemstra KA, Corssmit EP, van der Klaauw AA, Romijn JA, Smit JW. Quality of life in cured patients with differentiated thyroid carcinoma. J Clin Endocrinol Metab. 2008;93(1):200-203. 
67. Tagay S, Herpertz S, Langkafel M, et al. Health-related quality of life, anxiety and depression in thyroid cancer patients under short-term hypothyroidism and TSH-suppressive levothyroxine treatment. Eur J Endocrinol. 2005;153(6):755-763.

68. Vigario Pdos S, Chachamovitz DS, Cordeiro MF, et al. Effects of physical activity on body composition and fatigue perception in patients on thyrotropin-suppressive therapy for differentiated thyroid carcinoma. Thyroid. 2011;21(7):695-700.

69. Vigario Pdos S, Chachamovitz DS, Teixeira Pde F, Rocque Mde L, Santos ML, Vaisman M. Exercise is associated with better quality of life in patients on TSH-suppressive therapy with levothyroxine for differentiated thyroid carcinoma. Arq Bras Endocrinol Metabol. 2014;58(3):274-281.

70. Heemstra KA, Smit JW, Eustatia-Rutten CF, et al. Glucose tolerance and lipid profile in longterm exogenous subclinical hyperthyroidism and the effects of restoration of euthyroidism, a randomised controlled trial. Clin Endocrinol. 2006;65(6):737-744.

71. Hsieh CJ, Wang PW, Wang ST, et al. Serum leptin concentrations of patients with sequential thyroid function changes. Clin Endocrinol. 2002;57(1):29-34.

72. Rezzonico J, Niepomniszcze H, Rezzonico M, Pusiol E, Alberto M, Brenta G. The association of insulin resistance with subclinical thyrotoxicosis. Thyroid. 2011;21(9):945-949.

73. Horne MK, Singh KK, Rosenfeld KG, et al. Is thyroid hormone suppression therapy prothrombotic? J Clin Endocrinol Metab. 2004; 89(9):4469-4473.

74. Botella-Carretero JI, Prados A, Manzano L, et al. The effects of thyroid hormones on circulating markers of cell-mediated immune response, as studied in patients with differentiated thyroid carcinoma before and during thyroxine withdrawal. Eur J Endocrinol. 2005;153(2):223-230.
75. Smit JW, Eustatia-Rutten CF, Corssmit EP, et al. Reversible diastolic dysfunction after long-term exogenous subclinical hyperthyroidism: a randomized, placebo-controlled study. J Clin Endocrinol Metab. 2005;90(11):6041-6047.

76. Biondi B, Fazio S, Carella C, et al. Control of adrenergic overactivity by beta-blockade improves the quality of life in patients receiving long term suppressive therapy with levothyroxine. J Clin Endocrinol Metab. 1994;78(5):1028-1033

77. Gullu S, Altuntas F, Dincer I, Erol C, Kamel N. Effects of TSHsuppressive therapy on cardiac morphology and function: beneficial effects of the addition of beta-blockade on diastolic dysfunction. Eur $J$ Endocrinol. 2004;150(5):655-661.

78. Chan FK, Tiu SC, Choi KL, Choi CH, Kong AP, Shek CC. Increased bone mineral density in patients with chronic hypoparathyroidism. $J$ Clin Endocrinol Metab. 2003;88(7):3155-3159.

79. Mucsi I, Hercz G. Relative hypoparathyroidism and adynamic bone disease. Am J Med Sci. 1999;317(6):405-409.

80. Mikosch P, Obermayer-Pietsch B, Jost R, et al. Bone metabolism in patients with differentiated thyroid carcinoma receiving suppressive levothyroxine treatment. Thyroid. 2003;13(4):347-356.

81. Taimela E, Taimela S, Nikkanen V, Irjala K. Accelerated bone degradation in thyroid carcinoma patients during thyroxine treatment, measured by determination of the carboxyterminal telopeptide region of type I collagen in serum. Eur J Clin Chem Clin Biochem. 1994;32(11):827-831.

82. Mikosch P, Igerc I, Kudlacek S, et al. Receptor activator of nuclear factor kappaB ligand and osteoprotegerin in men with thyroid cancer. Eur J Clin Invest. 2006;36(8):566-573.

83. Mikosch P, Kerschan-Schindl K, Woloszczuk W, et al. High cathepsin $\mathrm{K}$ levels in men with differentiated thyroid cancer on suppressive L-thyroxine therapy. Thyroid. 2008;18(1):27-33.
Pragmatic and Observational Research

\section{Publish your work in this journal}

Pragmatic and Observational Research is an international, peer-reviewed open access journal that publishes data from studies designed to reflect more closely medical interventions in real-world clinical practice compared with classical randomized controlled trials (RCTs). The manuscript management system is completely online and includes a very quick and fair peer-review

\section{Dovepress}

system. Visit http://www.dovepress.com/testimonials.php to read real quotes from published authors.

Submit your manuscript here: https:/www.dovepress.com/pragmatic-and-observational-research-journal 